\title{
DESIGN OF A BEAM SIZE MONITOR USING FRESNEL ZONE PLATES
}

\author{
N. Nakamura, Y. Kamiya, T. Koseki, H. Takaki \\ Institute for Solid State Physics, University of Tokyo, Kashiwa, Chiba, Japan \\ N. Aoki, K. Nakayama, Toshiba Corporation, Yokohama, Japan
}

\begin{abstract}
Measuring electron-beam sizes is very important to estimate horizontal and vertical emittances in lowemittance rings. The beam size monitor using Fresnel zone plates (FZPs) has been designed for the Super-SOR ring (a new name of the VSX ring), which is a thirdgeneration synchrotron light source being planned by the University of Tokyo. The monitor system has a structure of a long-distance x-ray microscope using two FZPs and has an advantage of direct and real-time monitoring of the electron beam profile. The synchrotron radiation from the electron beam at a bending magnet is monochromatized by a double-crystal monochromator and the electron beam profile is magnified and imaged on an x-ray CCD camera by two FZPs. The spatial resolution is estimated to be about $1 \mu \mathrm{m}$ for the photon energy of $5 \mathrm{keV}$ and it is sufficiently high to measure the horizontal and vertical beam sizes, $14 \mu \mathrm{m}$ and $3.6 \mu \mathrm{m}$ at minimum, respectively. The design of the new beam profile monitor using Fresnel zone plates is presented in this paper.
\end{abstract}

\section{INTRODUCTION}

In the Super-SOR ring[1], the electron beam with extremely small sizes of $3.6 \mu \mathrm{m}$ (vertical) and $13.8 \mu \mathrm{m}$ (horizontal) can be stored. Therefore, measurement of the electron-beam size is considered as an important study item. The method utilizing a laser-Compton phenomenon is known as the method of beam-size measurement with nanometer-order accuracy[2]. However, it is expected that $\mathrm{x}$-ray imaging optics using new optical elements can directly monitor the electron-beam profile in a nondestructive manner.

Fresnel zone plates (FZPs) have been used for a x-ray microscope, and can focus $\mathrm{x}$-ray beams with the energy of less than $8 \mathrm{keV}[3]$. Also, the spatial resolution, which depends on the outermost-zone width of FZPs, is capable of being less than $100 \mathrm{~nm}$ [4]. We have tried to apply the FZPs to the beam size monitor and designed the x-ray optics and the other components. In this paper, the results of designing the new beam size monitor using FZPs are summarized.

\section{PRINCIPLE}

\subsection{Electron beam and synchrotron radiation of the Super-SOR ring}

The Super-SOR ring has 28 bending magnets (BMs) including 8 half BMs and one of the SR beam-lines equipped at the BMs can be used for the beam size monitor. The basic parameters of the Super-SOR ring are listed in Table 1.

Table 1. Basic parameters of the Super-SOR ring

\begin{tabular}{l|c|c|c}
\hline & $\begin{array}{c}\text { Ultra-low } \\
\text { emittance } \\
\text { mode }\end{array}$ & \multicolumn{2}{|c}{ Low-emittance mode } \\
\hline Energy $\mathrm{E}_{\mathrm{e}}[\mathrm{GeV}]$ & 1.0 & 1.0 & 1.6 \\
Emittance $\varepsilon_{0}[\mathrm{~nm} \mathrm{rad}]$ & 0.76 & 2.20 & 5.62 \\
Energy spread & $4.88 \mathrm{E}-04$ & $4.88 \mathrm{E}-04$ & $7.81 \mathrm{E}-04$ \\
Beam size $/$ divergence & & & \\
$\quad$ at the center of BM* & $13.8 / 63.4$ & $37.3 / 69.0$ & $59.6 / 110.3$ \\
Horizontal $[\mu \mathrm{m} / \mu \mathrm{rad}]$ & $3.6 / 2.1$ & $12.8 / 1.7$ & $20.5 / 2.7$ \\
Vertical $[\mu \mathrm{m} / \mu \mathrm{rad}]$ & 500.1 & 500.1 & 500.1 \\
RF frequency $\mathrm{f}_{\mathrm{RF}}[\mathrm{MHz}]$ & 2.04 & 2.90 & 4.17 \\
Bunch length $\sigma_{z}[\mathrm{~mm}]$ & \multicolumn{3}{|c}{} \\
* $1 \%$ coupling is assumed for both modes in this table.
\end{tabular}

The SR brilliance at the BM has a peak near the critical wavelength $\lambda_{\mathrm{c}}[\mathrm{nm}]$ given by

$$
\lambda_{\mathrm{c}}=1.86 /\left(\mathrm{E}_{\mathrm{e}}^{2} \mathrm{~B}\right)
$$

where $E_{e}[\mathrm{GeV}]$ is an electron beam energy and $\mathrm{B}[\mathrm{T}]$ is a magnetic field at the BM. The Super-SOR ring has two operation modes: the ultra-low emittance mode and the low emittance mode. At the former mode, the magnetic field is $1.092 \mathrm{~T}$ and the critical wavelength is $1.71 \mathrm{~nm}$, corresponding to the energy of $726 \mathrm{eV}$. Since a specific wavelength $\lambda$ is selected for the monitor, it is necessary to design the optics, considering the divergence of a single SR photon $\sigma_{\mathrm{SR}}[\mathrm{rad}]$ written by

$$
\sigma_{\mathrm{SR}}=0.565\left(\lambda / \lambda_{\mathrm{c}}\right)^{0.425} / \gamma
$$

where $\lambda_{\mathrm{c}}$ is the critical wavelength and $\gamma$ is the Lorentz factor.

\subsection{Fresnel Zone Plate}

Basic characteristics of a Fresnel zone plate is described here. The radius of $n$-th zone of the FZP is expressed by

$$
\mathrm{r}_{\mathrm{n}}=(\mathrm{nf} \lambda)^{1 / 2}
$$

where $\mathrm{f}$ is the focal length. The focal length for the $\mathrm{m}$-th order diffraction, $f_{m}$, is derived from equation (1) as follows:

$$
\mathrm{f}_{\mathrm{m}}=\mathrm{f}_{1} / \mathrm{m}=\mathrm{r}_{1}^{2} /(\mathrm{m} \lambda)
$$


The spatial resolution $\delta_{\mathrm{m}}$, the transverse size of a pointsource image for the $\mathrm{m}$-th order diffraction on the focal plane, is given by

$$
\delta_{\mathrm{m}}=1.22 \Delta \mathrm{r}_{\mathrm{N}} / \mathrm{m},
$$

where the suffix $\mathrm{N}$ is the total number of zones and $\Delta \mathrm{r}_{\mathrm{N}}$ is the width of the outermost zone.

\subsection{Imaging Optics}

In the beam size monitor, the imaged object is the cross-sectional profile of the electron-beam. The imaging optics is designed so that the required spatial resolution can be obtained at the focal point of each FZP.

The X-ray imaging optics using two FZPs has a structure of a long-distance microscope, as shown in Fig. 1. In order to ensure a sufficient spatial resolution, the SR has to uniformly irradiate the surface of the condenser zone plate (CZP) which is the first FZP.

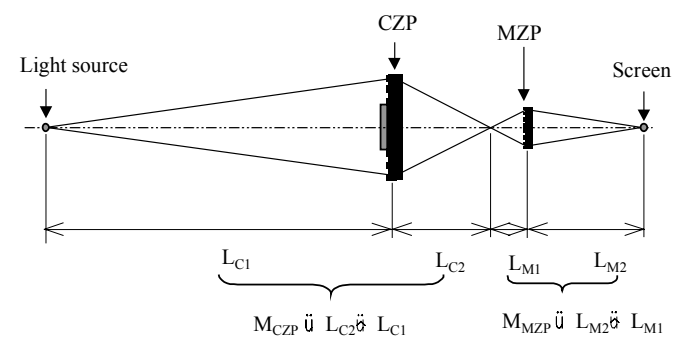

Figure 1: Layout of $x$-ray imaging optics

From equation (5) and $\Delta r_{N}=f \lambda / 2 r_{N}(N>>1)$, the spatial resolution for the 1-st order diffraction is given by

$$
\delta_{1}=1.22 \Delta \mathrm{r}_{\mathrm{N}}=1.22 \lambda \mathrm{f} /\left(2 \mathrm{r}_{\mathrm{N}}\right) .
$$

In Fig. 1, the distance $\mathrm{L}_{\mathrm{C} 2}$ from the CZP to the focal point is expressed by

$$
\mathrm{L}_{\mathrm{C} 2}=1 /\left(1 / \mathrm{f}-1 / \mathrm{L}_{\mathrm{C} 1}\right) .
$$

Since $\mathrm{L}_{\mathrm{C} 2}$ is nearly equal to the focal length $\mathrm{f}$ of the CZP on the condition of $L_{C 1} \gg f$, the magnification of the beam image $\mathrm{M}_{\mathrm{CZP}}$ by the CZP is given by

$$
\mathrm{M}_{\mathrm{CZP}} \cong \mathrm{f} / \mathrm{L}_{\mathrm{C} 1}
$$

Therefore, using equations (6) and (8), the spatial resolution at the SR source point on the electron-beam orbit, $\delta_{1}(0)$, can be expressed as follows:

$$
\delta_{1}(0)=\delta_{1} / \mathrm{M}_{\mathrm{CZP}} \cong 0.61 \lambda \mathrm{L}_{\mathrm{Cl}} / \mathrm{r}_{\mathrm{N}}
$$

Considering equation (2) for the SR divergence $\sigma_{\mathrm{SR}}$, the radius $r_{\mathrm{N}}$ of the CZP should be $\mathrm{L}_{\mathrm{Cl}} \sigma_{\mathrm{SR}}$ or less so that the SR uniformly irradiates the whole CZP surface. On the condition of $\mathrm{r}_{\mathrm{N}}=\mathrm{L}_{\mathrm{C} 1} \sigma_{\mathrm{SR}}$, the spatial resolution can be expressed by the following equation:

$$
\delta_{1}(0)=0.61 \lambda / \sigma_{\text {SR }}=1.08 \lambda^{0.575} \lambda_{\mathrm{c}}^{0.425} \gamma .
$$

Figure 2 shows the spatial resolution calculated from equation (10). From the results, it is clear that use of the SR with a higher energy is effective for obtaining a higher spatial resolution of the monitor.

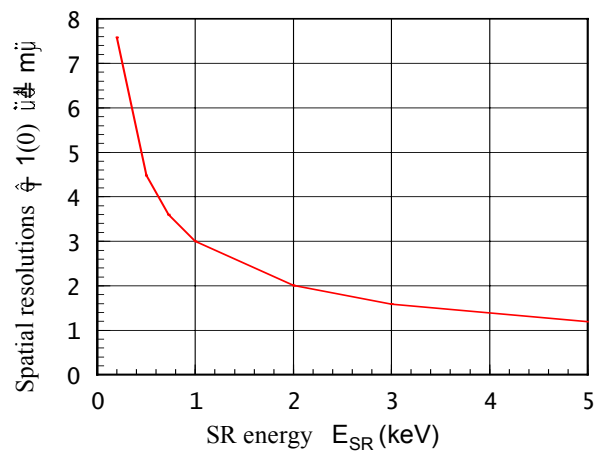

Figure 2: Spatial resolution of equation (10) as a function of the SR energy.

\section{DESIGN OF MONITORING SYSTEM}

Figure 3 shows the schematic view of the beam size monitor. The parameters of the optical elements are decided so that the image of the electron beam can be measured with spatial resolution of about $1 \mu \mathrm{m}$ for the ultra-low emittance mode in the Super-SOR ring. Here, the SR energy used for the monitor is set to $5 \mathrm{keV}$, considering the specifications of usually available FZPs.

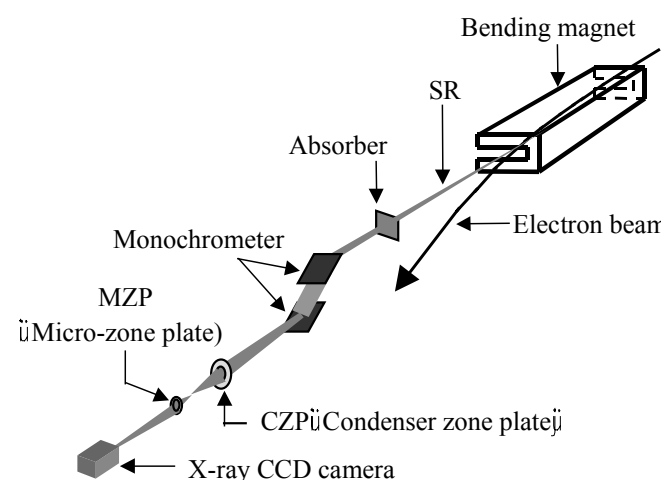

Figure 3: Schematic view of the beam size monitoring system for the Super-SOR ring.

The focal length of FZPs depends on the wavelength of the SR radiation, as shown in equation (3). The aberration caused by the energy bandwidth deteriorates the quality of the profile image. In order to avoid the effect of the aberration, the SR should be monochromatized before it arrives at the CZP. The spectral resolution $R(=E / \Delta E)$ is given by $R=D /(2 d)$, where $\mathrm{D}$ is the diameter of the CZP and $\mathrm{d}$ is the focus spot 
diameter[5]. Thus the necessary spectral resolution after monochromatizing is written by

$$
\mathrm{R}=\mathrm{E} / \Delta \mathrm{E}=\mathrm{D} /\left(2 \mathrm{~d}_{\mathrm{el}} \mathrm{M}_{\mathrm{CZP}}\right)
$$

where $d_{e l}$ is the minimum size of the electron beam and $\mathrm{M}_{\mathrm{CZP}}$ is the magnification of the CZP. The minimum size of the electron beam is expected to be about $3 \mu \mathrm{m}$. When the CZP with the diameter of $3 \mathrm{~mm}$ is used and the image size is reduced by a factor of 10 at the focal point of the $\mathrm{CZP}$, the monochromator with the spectral resolution of $5 \times 10^{3}$ is needed. This spectral resolution is easily achieved using a usual monochromator with two silicon crystals.

Since the electron-beam divergence is negligibly small in the Super-SOR ring, the divergence of a single SR photon is equal to that of the SR beam and it is calculated to be $0.127 \mathrm{mrad}$ from equation (2). The maximum diameter of usually available FZPs is about $3 \mathrm{~mm}$. Therefore, the distance from the SR source point to the CZP should be set to $12 \mathrm{~m}$ or more on the condition that $\mathrm{r}_{\mathrm{N}}$ is smaller than or equal to $\mathrm{L}_{\mathrm{C} 1} \sigma_{\mathrm{SR}}$.

Figure 4 shows the schematic view of the x-ray optics in the beam size monitoring system. The specifications of the optical elements including two FZPs are summarized in Table 2 .

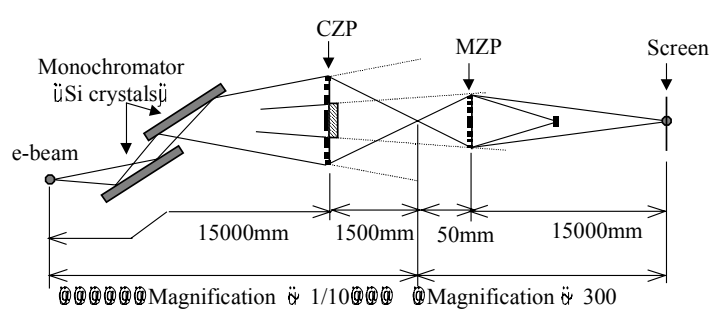

Figure 4: Schematic view of the x-ray optics in the beam size monitoring system of the Super-SOR ring.

Table 2: Specifications of the optical elements in the beam size monitor of the Super-SOR ring

\begin{tabular}{|c|c|c|}
\hline Fresnel zone plate & $(\mathrm{CZP})$ & $(\mathrm{MZP})$ \\
\hline Diameter & $3.0 \mathrm{~mm}$ & $100 \mu \mathrm{m}$ \\
\hline Total number of zones & 6671 & 202 \\
\hline Focal length @ $\mathrm{E}=5 \mathrm{keV}$ & $1360 \mathrm{~mm}$ & $49.89 \mathrm{~mm}$ \\
\hline Outermost zone width & $112 \mathrm{~nm}$ & $124 \mathrm{~nm}$ \\
\hline $\begin{array}{l}\text { Monochrometer } \\
\text { Spectral resolution }(\mathrm{E} / \Delta \mathrm{E}\end{array}$ & \multicolumn{2}{|l|}{$10^{4}-10^{5}$} \\
\hline $\begin{array}{l}\text { X-ray CCD camera (direct } \\
\text { Spatial resolution }\end{array}$ & X-ray CCD camera (direct incidence type) & type) \\
\hline
\end{tabular}

The total length and the arrangement of the monitoring system is decided, taking account of some restrictions of the SR beam line in the Super-SOR ring. In the optics, after the image of the electron beam is reduced by a factor of 10 by the CZP, it is 300 times magnified by the micro zone plate (MZP). Finally, the 30-times magnified image of the electron-beam can be obtained on the screen or the $x$-ray CCD camera. From equation (9), the spatial resolution of the $\mathrm{X}$-ray optics is estimated to be $1.37 \mu \mathrm{m}$. Therefore, the imaging camera needs the spatial resolution of less than $40 \mu \mathrm{m}$.

\section{DISCUSSION}

\subsection{Quality of profile image}

The quality of the beam profile image basically depends on spatial resolution and contrast. In order to obtain high contrast, cutting off the scattering ray into the the CCD camera is important. In the optics, only the SR from the electron beam at the source point should be imaged on the camera. Therefore, the aperture is installed above the absorber to eliminate the scattered radiation.

\subsection{Real-time monitoring}

The minimum measurement time of the beam size depends on the x-ray photon density on the CCD camera and the sensitivity of the camera. The number of the $\mathrm{x}$ ray photon per second on the camera depends on the stored beam current and it is roughly estimated to be ranging from $10^{4}$ to $10^{6}$ for the Super-SOR ring. When a direct incidence type of X-ray CCD camera is used, the number of the photons per pixel per second is expected to be $10^{2}$ to $10^{4}$. Accordingly the accumulation time of 0.01 to 1 second is needed in order to obtain a clear image. The readout time of the CCD camera is desired to be less than the accumulation time.

\section{CONCLUSION}

The new beam size monitor using Fresnel zone plates has been designed for the Super-SOR ring. It is concluded that the monitor can obtain the direct image of the electron-beam profile with the spatial resolution of about $1 \mu \mathrm{m}$. The conceptual design of the monitor is almost finished, and the engineering design and the construction of the test monitor system will be started soon.

\section{REFERENCES}

[1] K. Harada et al., These proceedings.

[2] T.Shintake, et al., Proc. of the 15th Int. Conf. on High Energy Accelerators, July, 20-24,1992.

[3] A. Ozawa, et al., Microelectronic Engineering 35, pp525-529, 1997.

[4] H. Rarback, et al., Rev. Sci. Instrum.59 (1), pp52-59, Jan., 1988.

[5] Xu Chaoyin, et al., Nucl. Instrum. Methods, A308, pp645-648, 1991. 